\title{
Transcranial direct current stimulation of the leg motor cortex enhances coordinated motor output during walking with a large inter-individual variability.
}

Edwin H. F. van Asseldonk ${ }^{1}$ and Tjitske A. Boonstra1*

${ }^{1}$ Department of Biomechanical Engineering, University of Twente, MIRA institute for biomechanical technology and technical medicine, $7500 \mathrm{AE}$, Enschede, The Netherlands

${ }^{*}$ T.A. Boonstra is now with Kennedy Krieger Institute, Baltimore, MD, United States and Department of Neuroscience, The Johns Hopkins University School of Medicine, United States

\section{Corresponding author:}

dr. Edwin H.F. Van Asseldonk

Laboratory for Biomechanical Engineering, MIRA institute for biomechanical technology and technical medicine, University of Twente, Faculty of Engineering Technology, PO Box 217, $7500 \mathrm{AE}$ Enschede, the Netherlands

Phone: +31 534892896 Fax: +31534893695 Email: e.h.f.VanAsseldonk@utwente.nl

Part of this work has been published in abstract form in the proceedings of the International Conference on Neurorehabilitation 2014 in Aalborg. 


\section{Abstract}

\section{Background}

Transcranial direct current stimulation (tDCS) can augment force generation and control in single leg joints in healthy subjects and stroke survivors. However, it is unknown whether these effects also result in improved force production and coordination during walking and whether electrode configuration influences these effects.

\section{Objective}

We investigated the effect of tDCS using different electrode configurations on coordinated force production during walking in a group of healthy subjects and chronic stroke survivors.

\section{Methods}

Ten healthy subjects and ten chronic stroke survivors participated in a randomized double-blinded crossover study. Subjects walked on an instrumented treadmill before and after 10 minutes of uni-hemispheric, (UNI) dual-hemispheric (DUAL) or sham tDCS applied to the primary motor cortex.

\section{Results}

tDCS responses showed large inter-individual variability in both subject populations. In healthy subjects tDCS enhanced the coordinated output during walking as reflected in an increased positive work generation during propulsion. The effects of DUAL tDCS were clearer but still small $(4.4 \%$ increase) compared to UNI tDCS (2.8\% increase). In the chronic stroke survivors no significant effects of tDCS in the targeted paretic leg were observed.

\section{Conclusions}

tDCS has potential to augment multi-joint coordinated force production during walking. The relative small contribution of the motor cortex in controlling walking might explain why the observed effects are rather small. Furthermore, a better understanding of the inter-individual variability is needed to optimize the effects of tDCS in healthy but especially stroke survivors. The latter is a prerequisite for clinical applicability.

Keywords: direct current stimulation, walking, stroke rehabilitation, motor cortex, lower extremities 


\section{Introduction}

Transcranial direct current stimulation (tDCS) is a non-invasive, painless and easy to apply brain stimulation technique that modulates the excitability of brain pathways, e.g., from the cortex to the muscles (corticomotor excitability) and may induce cortical plasticity [1-3]. The interest in tDCS has grown rapidly over the last years and many studies have assessed and tried to optimize its neurophysiological and behavioral effects on (the recovery of) the motor system. In this endeavor, the primary focus was on understanding effects in the upper extremities and only very limited studies were performed in the lower extremities.

Still, studies have shown that anodal tDCS applied over the leg motor cortex can influence the corticomotor excitability of different structures that are considered to play a role in the control of walking [4-6]. Also, tDCS leads to an increase in maximal voluntary pinch force, generated by the toes [7]. Additionally, it can facilitate subcortical structures [8], as it accelerated automatic postural responses which arise from subcortical structures. Whether all these changes will also result in an improved coordination during walking is largely unknown.

The ability to influence the motor coordination during walking would be of special importance for those people who suffer from gait deficits, for instance resulting from a stroke. That is, in stroke survivors, a decreased ability to generate sufficient propulsion with the paretic leg is one of the main causes for the slow walking speed [9] and patients use different strategies to compensate for this deficit (i.e. swing phase propulsion, circumduction) [10]. To improve the lower limb coordination of stroke patients, some studies have assessed the effects of anodal tDCS over the lesioned hemisphere on the force production and motor control in a single leg joint. Anodal stimulation resulted in an increased paretic knee extensor force generation $[11,12]$ and improved learning of a motor skill task with the paretic ankle [13]. Recently, a study showed that effects of tDCS in the lower extremities are not solely at the single joint level, but could also improve the performance on a functional task. Tahtis and colleagues [14] showed that tDCS resulted in a decrease of the time needed to complete the Timed-Up-and-Go-test (TUG), which consists of standing up, walking $3 \mathrm{~m}$, turning, walking back and sitting down. Although this study shows the potential of tDCS to influence tasks that require 
coordinated lower limb action, it was not reported whether the observed decrease in time could be attributed to a change in gait (speed) and if so how gait coordination was changed.

Moreover, the location of the cortical representation between the upper and lower extremities differs (i.e., the leg lies on the wall of the central fissure instead of in a gyrus). The latter indicates that different electrode configurations might be needed to optimize the electric field and/or electric field direction for the cortical leg representation [15]. In the standard protocols one electrode is placed over the area of interest and the other over the forehead. Recent experimental studies have demonstrated that effects are enhanced when placing one electrode over the target area and the other electrode over the same area on the contralateral side, so called dual-hemispheric stimulation $[16,17]$. Moreover, it has been hypothesized that dual-hemispheric stimulation normalizes the disturbed interhemispheric inhibition, as observed in stroke survivors [17-19].

The aim of this paper was to assess the effect of tDCS using different electrode configurations on the coordinated motor output in healthy subjects as well as stroke survivors. The contribution of the coordinated output of the muscles to forward propulsion and positive external work was quantified using the anterioposterior ground reaction forces [20,21]. As different spinal and supra-spinal structures (among which the motor cortex) are involved in the control of walking and their contribution could depend on attentional and energetic demands [22] we also investigated the influence of walking speed on the effects of tDCS in the group of healthy subjects.

\section{Methods}

\section{Subjects}

Ten healthy young subjects (nine male; age range 18-25 years) and ten chronic stroke survivors participated in this study. The healthy subjects were included to investigate whether tDCS would influence a normal walking pattern and have an isolated effect on the targeted leg. As we did not intend to perform a direct comparison between healthy subjects and stroke survivors, there was no need to include age-matched controls.

The stroke survivors suffered from a hemiparesis secondary to a single and first-ever unilateral ischemic or haemorrhagic stroke (see Table 1 for overview of patient characteristics). They were at 
least 6 months post-stroke, were able to walk independently (Functional Ambulation Categories (FAC) Scale $>4$ ) but had a clear walking impairment (for instance step length asymmetry). Also, they were able to walk on a treadmill for 3 minutes and were able to adequately comprehend our instructions.

Exclusion criteria for the stroke survivors and healthy subjects were the presence of epilepsy, intracranial metal, unexplained headaches, (possibility of) pregnancy, depression, other orthopedic, neurological or cardiac disorders. To characterize the patient population, all patients were scored the Functional Ambulation Categories [23], 10-m walking test (10MWT) and the Motricity Index (MI) of the paretic leg [24].

The study was approved by the medical ethical research committee of the Medisch Spectrum Twente (Enschede, The Netherlands) and conformed to the Declaration of Helsinki. All participants gave their written informed consent prior to the start of the experiment.

INSERT TABLE 1 HERE

\section{Experimental Design}

The subjects participated in a double-blind, placebo-controlled cross-over design consisting of 3 sessions performed at least 1 week apart and occurring around the same time of the day. The conditions were randomized and counterbalanced between the subjects. In each session subjects received tDCS with different configurations: a) uni-hemispheric (UNI), b) dual-hemipheric (DUAL) or c) sham stimulation (SHAM).

\section{Apparatus and Recordings}

In each session, we recorded the kinematics and kinetics while subjects walked on an instrumented split-belt treadmill (Y-mill, Forcelink, Culemborg, The Netherlands). Each belt was equipped with force sensors that allowed measurement of the 6 DoF ground reaction forces (GRF) and torques. These forces were sampled with $1000 \mathrm{~Hz}$. Kinematics were recorded using an active optical tracking system (Visualeyez VZ4000, PTI, Burnaby, Canada). We applied frames with three LEDs to the feet, lower legs and upper legs. The optical data was sampled with a frequency of $100 \mathrm{~Hz}$. The kinetic and kinematic data were samples using VZSoft software (PTI, Burnaby, Canada), which synchronizes the data collections and stores the data for offline analysis. 


\section{Transcranial direct current stimulation}

Direct current stimulation was delivered using a Magstim DC-Stimulator PLUS (Magstim company, Whitland, UK) through 2 saline-soaked $(0.9 \% \mathrm{NaCl})$ sponge electrodes $(5 \times 7 \mathrm{~cm})$. To prevent electric shunting, great care was taken to moisturize the sponges to an appropriate degree such that during the application of the electrodes no saline connection would be formed between the two electrodes. For the stroke survivors we determined the hotspot along the mid-sagittal fissure eliciting the largest motor evoked potentials (MEP) in the tibialis anterior of the affected leg using a Magstim Rapid² TMS stimulator (Magstim Company, Whitland, UK) with a double-cone coil (diameter $110 \mathrm{~mm}$ ). For UNI tDCS the anode was placed over the lesioned hemisphere with the short edge of the rectangular electrode aligned with the mid-sagittal fissure and centered around the identified hotspot and the cathode over contralateral supraorbital region (see Figure 1). For DUAL tDCS the anode was again placed over the lesioned hemisphere and centered around the hotspot but the electrode was shifted laterally $1 \mathrm{~cm}$, whereas the cathode was placed similarly over the contralateral hemisphere. So the inter electrode distance was $2 \mathrm{~cm}$ and electrodes were positioned on opposite sides of the target. For SHAM tDCS the electrode configuration was randomly assigned to the participant (either UNI or DUAL) and was balanced across the subjects. For UNI and DUAL tDCS the motor cortex was simulated for 10 minutes with $2 \mathrm{~mA}$. For SHAM the current was ramped down after it was being ramped up and held at $2 \mathrm{~mA}$ for $30 \mathrm{~s}$.

INSERT FIGURE 1 HERE

For the healthy subjects, we targeted the non-preferred leg, meaning that we determined the hotspot eliciting the largest MEP in the tibialis anterior of the non-preferred leg and placed the anodal electrode over hemisphere contralateral to the non-preferred leg. We determined the preferred leg (and thus the non-preferred leg) using a combination of three tasks (leading leg when stepping up a platform, leg used to step out when pushed from behind, preferred leg to kick a ball) [25]. 


\section{Experimental Procedure}

Each session consisted of a baseline measurement (BASE) of treadmill walking, tDCS and two post measurements of treadmill walking after approximately 15 (POST1) minutes and 45 minutes (POST2) (see Figure 2). In between these measurements, so also when receiving the stimulation, subjects were comfortably seating in a dentist chair. During the baseline and post measurements stroke survivors walked for 3 minutes at their preferred speed. This speed was determined at the end of a period of familiarization with walking on the treadmill in the first session, and was kept constant for all other sessions. The healthy subjects walked for 3 minutes at $2.5 \mathrm{~km} / \mathrm{h}$ (SLOW) and for 2 minutes at $5 \mathrm{~km} / \mathrm{h}$ (NORMAL). The order of the speeds was randomized between the different measurements and sessions. Sessions were approximately 2.5 hours, including preparation and rest.

\section{INSERT FIGURE 2 HERE}

\section{Data analysis}

Ground reaction forces and the recorded marker positions were low-pass filtered with a $2^{\text {nd }}$ order recursive Butterworth filter with a cut-off frequency of $15 \mathrm{~Hz}$. The foot position was calculated from the frame with three LEDs attached to the foot. Additionally, we calculated the anterioposterior (AP) Centre of Mass (CoM) velocity from the anterioposterior ground reaction forces by using a method described by Donelan and colleagues [21] and subsequently we calculated the power generated on the CoM (external mechanical power) for each leg by multiplying the AP ground reaction forces below each foot with the AP CoM velocity [21]. All these signals were segmented into individual stride cycles using the heel strikes as derived from the vertical ground reaction forces from each belt.

Subsequently, we calculated the different variables for each step. As a measure for the coordinated motor output we determined the total propulsive impulse as the integral over time of all positive AP GRF for each individual leg (Imp prop). We derived the positive work performed by the AP GRF on the CoM by calculating the integral over time of all positive external mechanical power [21] for each leg (see Figure 3). Furthermore, we calculated some spatiotemporal gait variables (i.e., cycle time) to assess how potential changes in propulsion would influence the gait pattern. Cycle time was calculated as the time between two subsequent heel strikes and the step length was calculated as the distance between the trailing and leading foot at the moment of heel strike of the leading foot. For each trial we calculated 
an average value (generally over approximately 80-100 steps) and expressed this as a percentage change relative to the baseline value.

\section{Statistics}

To assess the effect of the applied tDCS in the stroke survivors we performed a linear mixed model analysis with STIMULATION (UNI, DUAL, SHAM) and TIME (POST1 and POST2) as fixed factors for each dependent variable. To account for the correlation between the repeated measurements within a subject, different intercepts were assumed for each subject by including the factor subject as random factor into the analysis. For the healthy subjects we performed a similar linear mixed model analysis but also included SPEED $(2.5 \mathrm{~km} / \mathrm{h}$ and $5.0 \mathrm{~km} / \mathrm{h})$ as a fixed factor. Alpha was set at 0.05 and a Bonferroni correction was used to correct for multiple corrections. For the statistical analysis we used IBM SPSS statistics, version 20.0.

\section{Results}

\section{Direct current stimulation enhances propulsion in healthy subjects}

Active tDCS resulted in an increase of the propulsive impulsive and an accompanying increase of the positive work on the CoM for the targeted non-preferred leg (see Figure 3 for a representative responder). For DUAL and UNI tDCS the increases were respectively $4.4 \%$ and $2.8 \%$ with respect to baseline (see Figure 4 and Table 2). This increase was significantly different compared to SHAM for DUAL tDCS ( $\mathrm{p}=0.004)$ and almost reached significance for UNI tDCS $(\mathrm{p}=0.072)$ (see Table 2).

\section{INSERT FIGURE 3 AND 4}

\section{INSERT TABLE 2}

For DUAL tDCS the larger propulsion also resulted in a significantly larger increase in non-preferred stance duration $(\mathrm{p}=0.003)$ and cycle time $(\mathrm{p}=0.002)$ compared to SHAM tDCS as well as UNI tDCS ( $p=0.009$ and $p=0.007$, respectively). For step length, DUAL tDCS resulted in a significant increase compared to UNI ( $p=0.026)$ and a strong trend towards improvement compared to SHAM $(p=0.066)$. An 
increase in cycle time for a particular speed implies that the stride length also increases. The increase was particularly evident for the slow walking speed (see Figure 4). For the normal walking speed, effects were often minor but generally in the same direction as those found for the slow walking speed. Despite this difference in magnitude of response, we did not find any significant interaction effect between STIM and SPEED for the gait parameters derived from the non-preferred leg. For the propulsive impulse of the preferred leg there was a significant interaction effect (see Figure 5), but here no main effect was observed. Post hoc tests turned out that only for the slow walking speed the increase after DUAL tDCS was significantly larger than in the other conditions. Subjects could show an increased propulsive impulse in both legs while they kept walking with the same speed as they also increased their breaking impulse, which absorbed the additional propulsive work.

\section{INSERT FIGURE 5}

There were no consistent differences between tDCS-induced effects at Post1 and Post2 as there was no main effect of TIME for any of the variables. This means that the observed effects at least lasted until the post 2 measurement approximately 45 minutes after the end of the stimulation.

\section{Inter-individual variability in tDCS-induced effects is large in healthy subjects}

Although many main effects of tDCS were demonstrated, there was considerable inter-individual variability in response to the different forms of tDCS. This was especially true for the variables derived from the recorded ground reaction forces (see Figure 4). For instance, DUAL tDCS results in an across subjects average increase of the propulsive impulse of $7.1 \%$ when walking slowly. Yet five subjects showed an increase between 14 and $21 \%$, two subject showed a minor increase between 0 and $5 \%$ and three subjects even showed a decrease between 3 and $8 \%$.

\section{Direct current stimulation does not enhance propulsion on the paretic side}

One of the stroke survivors held the rails during the walking trials, which affects the ground reaction forces and the resulting movements in an unpredictable way and therefore, we excluded this subject from our analysis. 
Stroke survivors showed a clear asymmetry between the propulsive impulse of their paretic leg and non-paretic leg (see Figure 3). They generated less propulsion with their paretic leg and compensated this with larger propulsion on their non-paretic leg. Direct current stimulation did not result in a significant improvement of the paretic propulsion ( $p=0.186$, see Table 3 and Figure 6$)$ and consequently also the positive work and step length were not improved $(\mathrm{p}=0.407$ and $\mathrm{p}=0.306$ respectively). Also the stroke survivors showed large inter-individual variability in their response to the direct current stimulation. As the sample size was rather small, we could not reliably relate the response to the stimulation to certain patient characteristics or lesion location. Additionally, we also found substantial intra-subject variability in response to different forms of active stimulation. Subjects showing the largest change for one form of active stimulation (UNI or DUAL tDCS) often showed opposite effects for the other form of active stimulation.

\section{INSERT TABLE 3 HERE}

\section{INSERT FIGURE 6 HERE}

Remarkably, direct current stimulation did result in a significant $(\mathrm{p}=0.034)$ albeit small improvement of the non-paretic positive work (UNI: $2.7 \pm 0.2$,DUAL: $2.5 \pm 0.2$, SHAM:-3.3 \pm 0.2 ) and a trend $(\mathrm{p}=0.094)$ towards a larger propulsive impulse. Post hoc tests revealed that the positive work for DUAL as well as UNI showed a trend towards improvement compared to SHAM ( $p=0.078$ and $p=0.066$ respectively).

No interaction effects between STIM and TIME were observed. TIME did have a significant main effect for some of the variables (cycle time, non-paretic stance duration), were the effects observed at POST2 were significantly larger than those at POST1. However, the observed differences were small $(<1.5 \%$ difference $)$.

\section{Discussion}

In this sham-controlled randomized study, we demonstrated that non-invasive electrical stimulation over the leg motor cortex can influence the coordinated motor output and spatiotemporal 
variables of walking in healthy subjects. It must be noted though, that there are large inter-individual differences in response to tDCS, with only $50 \%$ of the healthy subjects showing a clear beneficial effect. The tDCS effects were more pronounced when using a dual-hemispheric electrode montage compared to using a uni-hemispheric montage. For chronic stroke survivors the stimulation did not result in improved motor coordination on their paretic, targeted side and inter-individual differences in response were even larger in these subjects.

\section{tDCS effects show a large inter-individual variability}

Individual responses to tDCS varied widely. For instance, in the condition that showed the largest improvement in healthy subjects (dual-hemispheric stimulation during slow walking) only $50 \%$ of the subjects showed a distinct anticipated response and conversely, $30 \%$ showed a clear opposite effect in the propulsive impulse. Similar variability in responses of the healthy subjects was observed for other variables and in other conditions. Although most studies on tDCS-induced effects only report group effects and neglect inter-individual difference [26], some recent papers have focused on this inter-individual variability $[27,28]$. Both studies investigated the variability of tDCS-induced changes in corticomotor excitability of a hand muscle in a group of over 50 persons. They showed that approximately $50 \%$ responded as expected and showed a facilitatory effect of the stimulation. A previous study of Madhavan and colleagues [29] showed that, in response to anodal stimulation over the leg cortex, $60 \%$ of the subjects showed an increase of corticomotor excitability in leg muscle. These "response rates" are in accordance with the findings of our study.

Variability in responses was even more pronounced in the stroke survivors. This could be due to a variety of reasons. First,, the stroke survivors differed in the brain region that was affected by the stroke. As these different brain regions play different roles in the control of walking, this will likely result in different walking impairments, which could differ in their susceptibility to tDCS induced effects. Second, variability could arise from differences in the degree of damage to the stimulated area (primary motor cortex) or downstream brain regions involved in conveying signals from the motor cortex to the leg muscles. Third, recovery of walking function resulting from rehabilitation is driven by cortical reorganization, where brain areas take over (some of the) tasks of the damaged brain areas [30]. It is very well possible that this cortical reorganization alters the effect of tDCS, as in the stroke 
survivors other pathways become involved in the generation of the locomotor pattern, compared to the healthy controls and these pathways may not be equally effected by tDCS. In sum, there are different causes for the observed increased variability in tDCS induced effects and more insight is needed into the relation between these variability sources and tDCS-induced effects to be able to determine which stroke survivors could potentially benefit from tDCS [31].

\section{tDCS improves the coordinated motor output during walking}

Previous studies showed that tDCS over the leg motor cortex can influence force generation and control in single leg joints in healthy subjects [7] as well as chronic stroke survivors [11-13]. At least for healthy subjects, our results extend these findings as we demonstrated (despite the large variability) that $\mathrm{tDCS}$ can result in an increased force production during the push off phase of walking, such that the propulsive impulse increases. The increased propulsion was accompanied by larger strides and longer cycle time. This longer cycle time is probably a mere consequence of the increased stride length as subjects had to slow down their walking cycle to keep walking at the imposed constant treadmill speed. It is likely that the same change in propulsion would have resulted in an increased walking speed when walking over ground. Hence, an increase in propulsive forces could explain the recently demonstrated faster completion of the TUG test after receiving DUAL tDCS in sub-acute stroke survivors [14].

\section{DUAL tDCS also induced behavioral effects on the non-targeted body side}

In the DUAL tDCS configuration, a cathode is placed over the motor cortex contralateral to the targeted area, where it is supposed to have an inhibitory effect on the non-targeted leg. However, we observed a significant increase in the amount of positive work and propulsive impulse of the non-targeted leg in respectively the stroke survivors (non-paretic leg) and healthy subjects (preferred leg).

There are several possible explanations for this finding. First, tDCS could have caused facilitation of ipsilateral pathways that travel to the non-targeted leg. That is, Madhavan and colleagues [29] showed that an increase in excitability of the contralateral pathways to the leg muscles after tDCS was 
accompanied by an increase in excitability of the ipsilateral pathway. Second, DUAL tDCS could have caused facilitation of the indirect pathways via subcortical structures to the lower leg muscles. Recently, Nonnekes and colleagues [8] have shown that tDCS over the motor cortex result can result in changes in excitability of subcortical structures, as evidenced by a decrease of the onset time of automatic postural responses in the tibialis anterior. Importantly, they showed that the decrease in onset times occurred in the targeted, as well as in the non-targeted leg. Third, the subjects might have altered the activation of the non-targeted leg in response to the induced effects in the targeted leg in order to restore the symmetry in walking. This explanation only holds for the healthy subjects, as in the stroke survivors no effects were observed in the targeted, paretic leg. For UNI tDCS, no significant effects on the non-targeted leg were observed, so apparently the strength and or the direction of the resulting electrical field did not result in changes in the polarization of neurons in the ipsilateral or indirect pathway in this configuration.

\section{Dual tDCS tended to have a larger effect on walking in healthy subjects}

Dual-hemisperic stimulation tended to have a larger effect on the walking pattern of the healthy subjects than uni-hemisperic stimulation; either only the dual-hemisperic stimulation resulted in a significant difference with sham stimulation and/or dual-hemisperic stimulation resulted in significantly larger changes than uni -hemisperic stimulation. To date, the potential of dual-hemisperic or bihemisperic stimulation has been mainly shown for the upper extremities where it was shown to have a beneficial effect for healthy subjects on motor learning [32] and for stroke survivors on motor performance [18], motor learning and retention [31,33] and motor recovery $[17,34]$. The few studies that directly compared the effects of dual-/ and uni-hemisperic stimulation showed larger beneficial effects for dual-hemispheric stimulation [32,35]. The additional effect of cathodal tDCS over the contralateral motor cortex is attributed to modulation of the inhibitory projections between the motor cortices of both hemispheres. This effectively results in a disinhibition of the ipsilateral cortex, that would add up to the already upregulating effect of the anodal stimulation [32]. For the lower extremities there is also evidence for the existence of this interhemisperic inhibition and its potential role in the control of movement $[5,13]$. For the lower extremities, there has been no direct comparison of different electrode configurations and this study has been the first to show larger effects for the 
dual-hemispheric configuration. By using a detailed anisotropic human head model [15], simulating the different electrode configurations, showed that the DUAL configuration had a stronger electric field in the targeted area (unpublished results). Hence, the observed differences between the UNI and DUAL configurations could be due to different electric field strengths in each condition. Still, the differences were small and only present in the healthy subjects.

\section{Effects of tDCS on gait are small}

tDCS of the leg motor cortex increased the propulsive force (on average) only by $4.4 \%$ (DUAL) and $2.8 \%(\mathrm{UNI})$, respectively, which is small compared to the reported effects in single leg joints. For example, Tanaka reported an increase in toe-pinch force of $20 \%$ in healthy subjects [7], whereas anodal tDCS over the lesioned hemisphere in stroke survivors resulted in increased knee extensor force of $13 \%$ [11] to $20 \%$ [12]. Hence, our results indicate that the effects found in single leg joints do not necessarily transfer to whole body ambulation. Yet, care should be taken in comparing average values. As already pointed out earlier, tDCS does not have a beneficial effect in all subjects. Especially, for smaller samples the across group average is strongly influenced by the number of responders in the sample.

Gait is an automated movement, largely controlled by subcortical structures and networks in the spinal cord and therefore we hypothesize that the here reported small effects on gait are due to the relative small contribution of the corticospinal pathway to walking movements, compared to a voluntary task. Our results also showed that the effects of tDCS were more pronounced for slower speeds, which might suggest that walking at or close to your preferred speed is even more automated and requires less cortical involvement [36].

\section{Reliability of outcome measures}

Not only did we observe considerable variability in response to real simulation, we also observed substantial variability in the kinetic variables, propulsive impulse and positive mechanical work, in the sham condition. This observed variability can be a mixture of fatiguing effects, habituation effects, susceptibility to placebo and reliability of the measure. We did our best to prevent fatiguing effects (allowing them rest in between walking trials) and habituation effects (providing them with a 
warming-up walking trial at the start of each session). Furthermore we expect that the reliability of these measures is good as the test-retest reliability of total external mechanical work (so positive and negative of both legs) was shown to be excellent in stroke survivors with an intra-class correlation (ICC) of 0.96 [37]. All in all, from the current results we cannot discern the contribution of the different factors to the observed variability. Still, the effects of real tDCS were sufficiently large to be revealed despite this large variability.

\section{Future directions}

In this study, we have shown that tDCS has the potential to facilitate the coordinated force generation during push-off, in a selection of subjects. Therefore, tDCS could be used as an adjuvant to other forms of therapy, e.g., to increase the walking performance during the therapy and induce long-lasting and clinically meaningful effects. However, before the technique becomes clinically available stimulation protocols need to be developed that result in a larger number of responders and a larger effect in these responders. Furthermore, we need to better understand the causes of the response variability and we need methods to identify those subjects who will benefit from tDCS,. Tackling the variability is one of the major challenges for the field of tDCS research. Although some causes of inter-individual differences have been pointed out (e.g., anatomical differences) [26] and several neurophysiological measures that can predict (part of) this variability have been identified [28,38], this variability still remains poorly understood. A better understanding can be achieved by identifying biomarkers (be it neurophysiological, imaging or behavioral) in individual participants in order to predict which participant will respond in what way (see for instance [39]).

\section{Acknowledgment}

This study was supported by a VENI grant (grant nr: 91610143) from The Netherlands Organisation for Scientic Research. The funding source had no direct involvement in conduction of the study. The authors want to thank Stan Verwer and Thijs Lohuis for their help in collecting the data and Bertine Fleerkotte, Jaap Buurke and Juliëtte Nijlant for their help in recruiting the stroke survivors. 


\section{References}

[1] Harris-Love M, Cohen L. Noninvasive cortical stimulation in neurorehabilitation: a review. Arch Phys Med Rehabil 2006;87:S84-93.

[2] Nitsche M, Paulus W. Excitability changes induced in the human motor cortex by weak transcranial direct current stimulation. J Physiol 2000;527 Pt 3:633-9.

[3] Nitsche M, Paulus W. Sustained excitability elevations induced by transcranial DC motor cortex stimulation in humans. Neurology 2001;57:1899-901.

[4] Jeffery DT, Norton JA, Roy FD, Gorassini MA. Effects of transcranial direct current stimulation on the excitability of the leg motor cortex. Exp Brain Res 2007;182:281-7.

[5] Jayaram G, Stinear J. The effects of transcranial stimulation on paretic lower limb motor excitability during walking. Journal of Clinical Neurophysiology : Official Publication of the American Electroencephalographic Society 2009;26:272-9.

[6] Kim CR, Kim D-Y, Kim LS, Chun MH, Kim SJ, Park CH. Modulation of cortical activity after anodal transcranial direct current stimulation of the lower limb motor cortex: a functional MRI study. Brain Stimulation 2012;5:462-7.

[7] Tanaka S, Hanakawa T, Honda M, Watanabe K. Enhancement of pinch force in the lower leg by anodal transcranial direct current stimulation. Exp Brain Res 2009;196:459-65.

[8] Nonnekes J, Arrogi A, Munneke MAM, van Asseldonk EHF, Oude Nijhuis LB, Geurts AC, et al. Subcortical structures in humans can be facilitated by transcranial direct current stimulation. PLoS ONE 2014;9:e107731.

[9] Nadeau S, Gravel D, Arsenault A, Bourbonnais D. Plantarflexor weakness as a limiting factor of gait speed in stroke subjects and the compensating role of hip flexors. Clin Biomech (Bristol, Avon) 1999;14:12535 .

[10] Chen G, Patten C, Kothari D, Zajac F. Gait differences between individuals with post-stroke hemiparesis and non-disabled controls at matched speeds. Gait Posture 2005;22:51-6.

[11] Tanaka S, Takeda K, Otaka Y, Kita K, Osu R, Honda M, et al. Single Session of Transcranial Direct Current Stimulation Transiently Increases Knee Extensor Force in Patients With Hemiparetic Stroke.

Neurorehabilitation and Neural Repair 2011;25:565-9.

[12] Sohn MK, Jee SJ, Kim YW. Effect of Transcranial Direct Current Stimulation on Postural Stability and Lower Extremity Strength in Hemiplegic Stroke Patients. Ann Rehabil Med 2013;37:759.

[13] Madhavan S, Weber KA, Stinear JW. Non-invasive brain stimulation enhances fine motor control of the hemiparetic ankle: implications for rehabilitation. Exp Brain Res 2011;209:9-17.

[14] Tahtis V, Kaski D, Seemungal BM. The effect of single session bi-cephalic transcranial direct current stimulation on gait performance in sub-acute stroke: A pilot study. Restor Neurol Neurosci 2014;32:52732.

[15] Rampersad SM. Simulation of stimulation: on modeling and measuring the effects of transcranial direction current stimulation on the human brain. PhD Thesis Radboud University, 2015.

[16] Mahmoudi H, Borhani Haghighi A, Petramfar P, Jahanshahi S, Salehi Z, Fregni F. Transcranial direct current stimulation: electrode montage in stroke. Disabil Rehabil 2011;33:1383-8.

[17] Lindenberg R, Renga V, Zhu LL, Nair D, Schlaug G. Bihemispheric brain stimulation facilitates motor recovery in chronic stroke patients. Neurology 2010;75:2176-84.

[18] Lefebvre S, Thonnard J-L, Laloux P, Peeters A, Jamart J, Vandermeeren Y. Single session of dual-tDCS transiently improves precision grip and dexterity of the paretic hand after stroke. Neurorehabilitation and Neural Repair 2014;28:100-10.

[19] Nowak D, Grefkes C, Ameli M, Fink G. Interhemispheric competition after stroke: Brain stimulation to enhance recovery of function of the affected hand. Neurorehabilitation and Neural Repair 2009;23:64156.

[20] Bowden MG, Balasubramanian CK, Neptune RR, Kautz SA. Anterior-posterior ground reaction forces as a measure of paretic leg contribution in hemiparetic walking. Stroke 2006;37:872-6.

[21] Donelan J, Kram R, Kuo A. Simultaneous positive and negative external mechanical work in human walking. J Biomech 2002;35:117-24.

[22] Clark DJ, Kautz SA, Bauer AR, Chen Y-T, Christou EA. Synchronous EMG activity in the piper frequency band reveals the corticospinal demand of walking tasks. Ann Biomed Eng 2013;41:1778-86.

[23] Holden M, Gill K, Magliozzi M, Nathan J, Piehl-Baker L. Clinical gait assessment in the neurologically impaired. Reliability and meaningfulness. Phys Ther 1984;64:35-40.

[24] Collin C, Wade D. Assessing motor impairment after stroke: a pilot reliability study 1990;53:576-9.

[25] De Ruiter CJ, De Korte A, Schreven S, de Haan A. Leg dominancy in relation to fast isometric torque production and squat jump height. Eur J Appl Physiol 2010;108:247-55.

[26] Horvath JC, Carter O, Forte JD. Transcranial direct current stimulation: five important issues we aren't discussing (but probably should be). Front Syst Neurosci 2014;8:2.

[27] López-Alonso V, Cheeran B, Río-Rodríguez D, Fernández-del-Olmo M. Inter-individual Variability in 
Response to Non-invasive Brain Stimulation Paradigms. Brain Stimulation 2014;7:372-80.

Wiethoff S, Hamada M, Rothwell JC. Variability in Response to Transcranial Direct Current Stimulation of the Motor Cortex. Brain Stimulation 2014;7:468-75.

[29] Madhavan S, Stinear JW. Focal and bidirectional modulation of lower limb motor cortex using anodal transcranial direct current stimulation. Brain Stimulation 2010;3:42-50.

[30] Belda-Lois J-M, Mena-del Horno S, Bermejo-Bosch I, Moreno JC, Pons JL, Farina D, et al. Rehabilitation of gait after stroke: a review towards a top-down approach. J Neuroengineering Rehabil 2011;8:66.

[31] Lefebvre S, Dricot L, Laloux P, Gradkowski W, Desfontaines P, Evrard F, et al. Neural substrates underlying stimulation-enhanced motor skill learning after stroke. Brain 2015;138:149-63.

[32] Vines B, Cerruti C, Schlaug G. Dual-hemisphere tDCS facilitates greater improvements for healthy subjects' non-dominant hand compared to uni-hemisphere stimulation. BMC Neurosci 2008;9:103.

[33] Lefebvre S, Laloux P, Peeters A, Desfontaines P, Jamart J, Vandermeeren Y. Dual-tDCS Enhances Online Motor Skill Learning and Long-Term Retention in Chronic Stroke Patients. Front Hum Neurosci 2013;6.

[34] Bolognini N, Vallar G, Casati C, Latif LA, El-Nazer R, Williams J, et al. Neurophysiological and behavioral effects of tDCS combined with constraint-induced movement therapy in poststroke patients. Neurorehabilitation and Neural Repair 2011;25:819-29.

[35] Lindenberg R, Nachtigall L, Meinzer M, Sieg MM, Floel A. Differential effects of dual and unihemispheric motor cortex stimulation in older adults. Journal of Neuroscience 2013;33:9176-83.

[36] Jahn K, Deutschländer A, Stephan T, Strupp M, Wiesmann M, Brandt T. Brain activation patterns during imagined stance and locomotion in functional magnetic resonance imaging. NeuroImage 2004;22:172231.

[37] Caty GD, Detrembleur C, Bleyenheuft C, Lejeune TM. Reliability of lower limb kinematics, mechanics and energetics during gait in patients after stroke. J Rehabil Med 2009;41:588-90.

[38] O'Shea J, Boudrias M-H, Stagg CJ, Bachtiar V, Kischka U, Blicher JU, et al. Predicting behavioural response to TDCS in chronic motor stroke. NeuroImage 2014;85 Pt 3:924-33.

[39] Bradnam LV, Stinear CM, Barber PA, Byblow WD. Contralesional hemisphere control of the proximal paretic upper limb following stroke. Cerebral Cortex 2012;22:2662-71. 


\section{Figures}

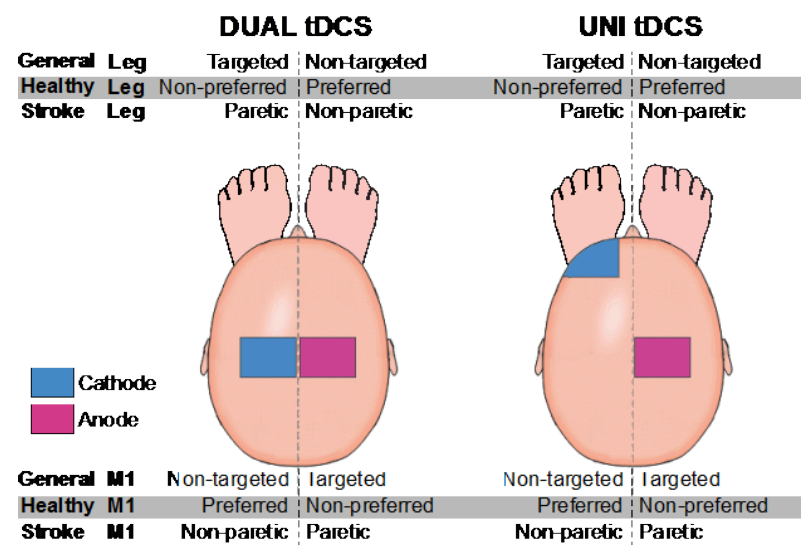

Figure 1. Schematic illustration of the electrode positions for the UNI and DUAL configuration for the healthy subjects and chronic stroke survivors. 


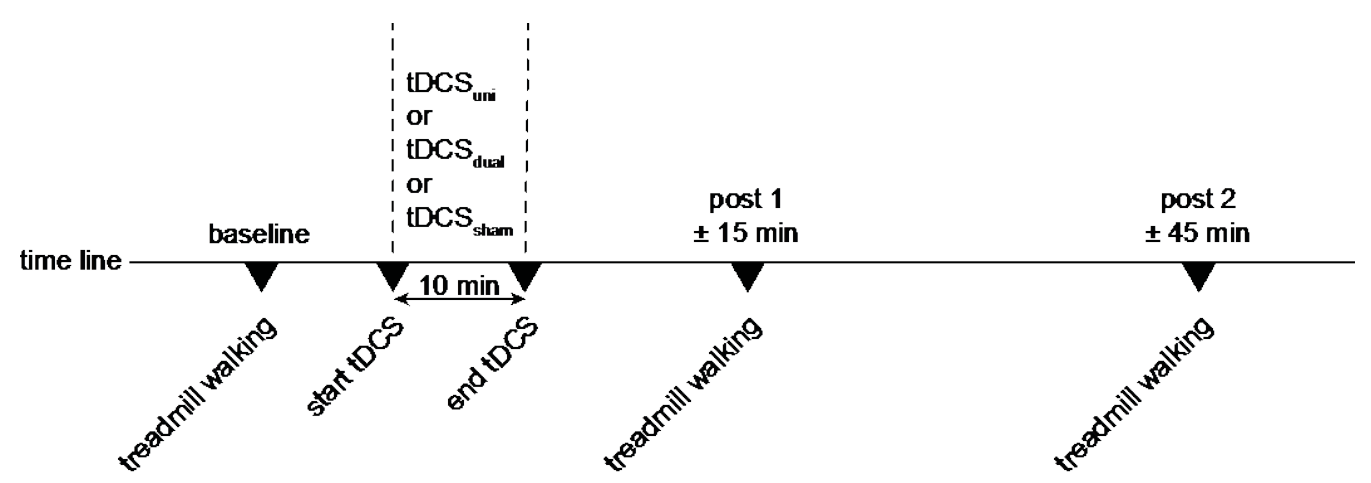

Figure 2. Schematic overview of the experimental procedure in each session. 


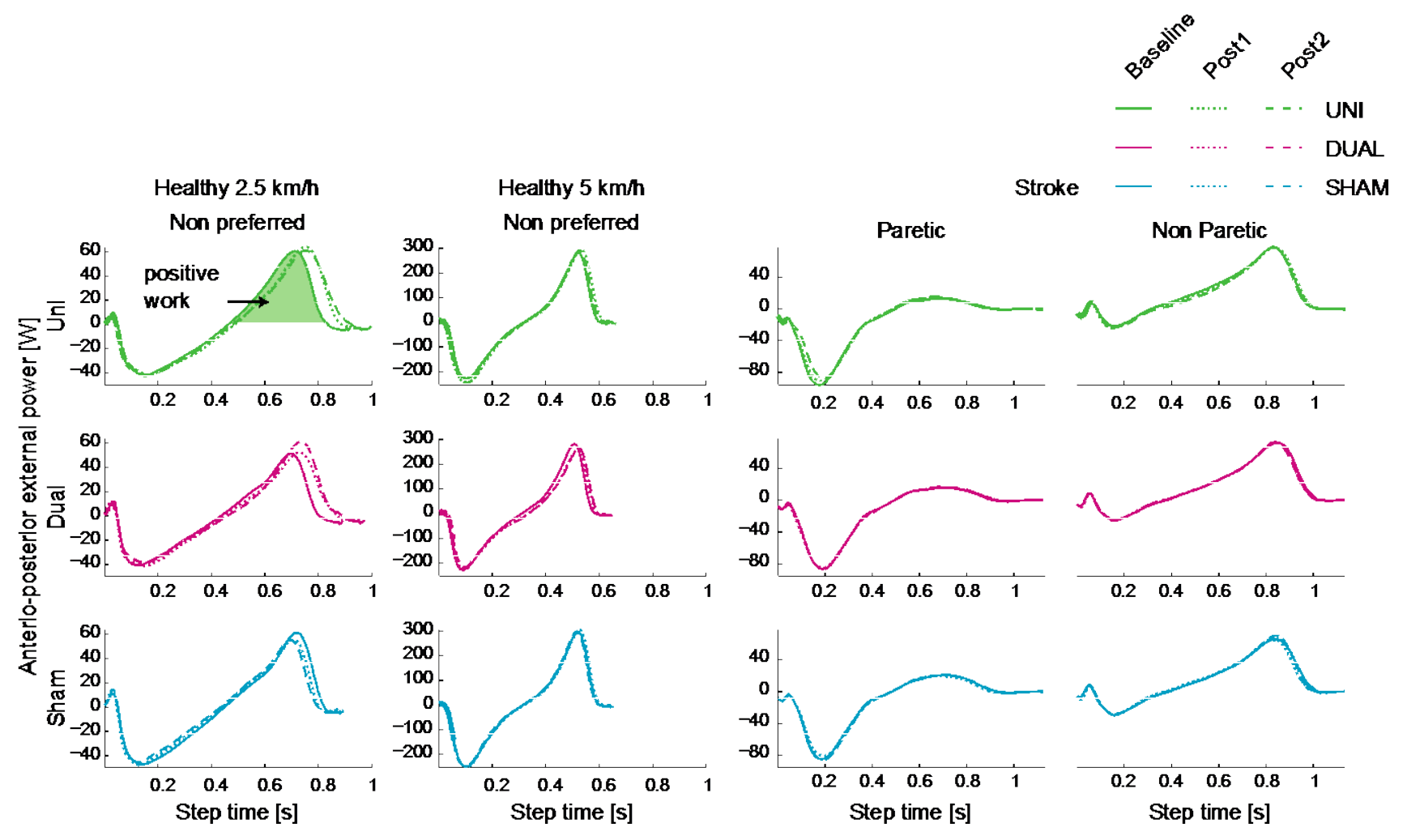

Figure 3. Representative examples of external power in anterio-posterior direction as a fuction of time for the non-preferred leg of a healthy subject walking at 2.5 and $5 \mathrm{~km} / \mathrm{h}$ and for the paretic and non-paretic leg of a chronic stroke survivor. The different rows indicate the response for the different stimulation modes and the different lines in the graph indicate the different measurements within one session. 

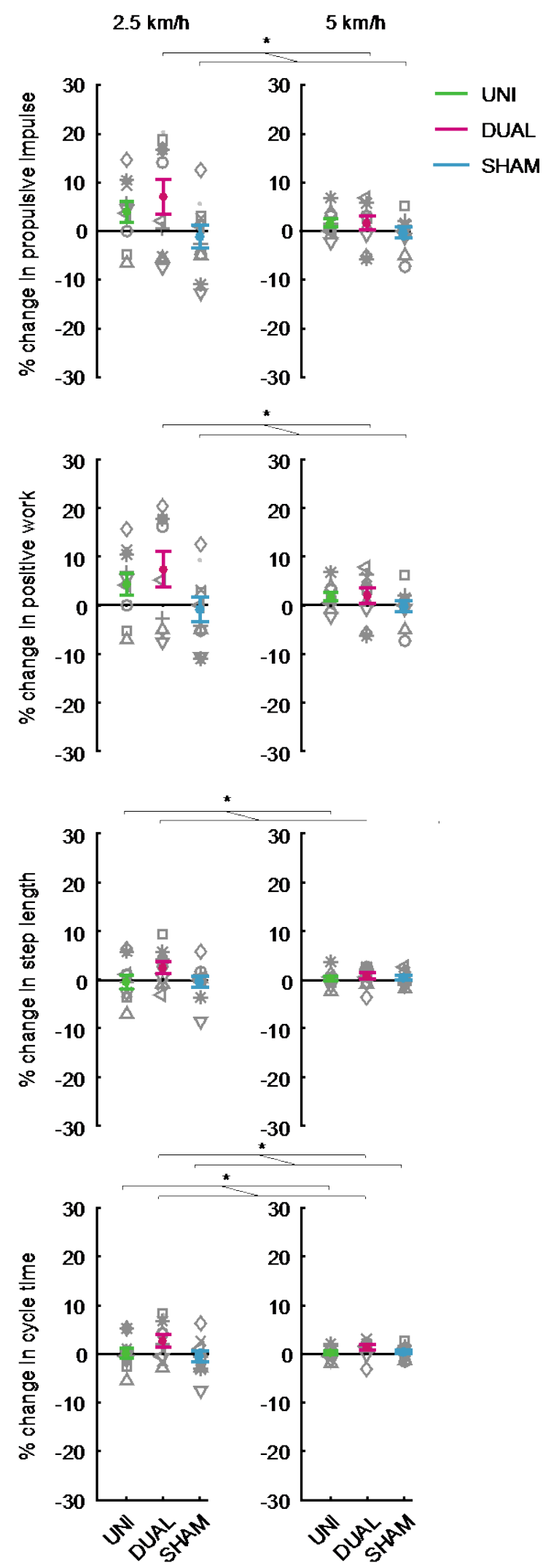

Figure 4. Group averages for the outcome variables for the non-preferred leg of healthy subjects expressed as a percentage change from the baseline values for the different experimental conditions. The thick dotted marker and error bars indicate the across subject averages and standard error of the mean. The gray markers indicate the individual responses (each subject has its own marker). 


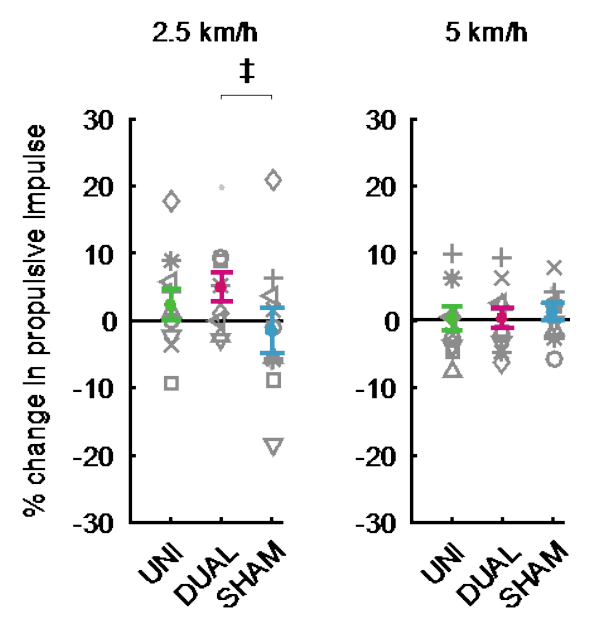

Figure 5. Group averages for the outcome variables for the preferred leg of healthy subjects expressed as a percentage change from the baseline values for the different experimental conditions. The thick dotted marker and error bars indicate the across subject averages and standard error of the mean. The gray markers indicate the individual responses (each subject has its own marker). 

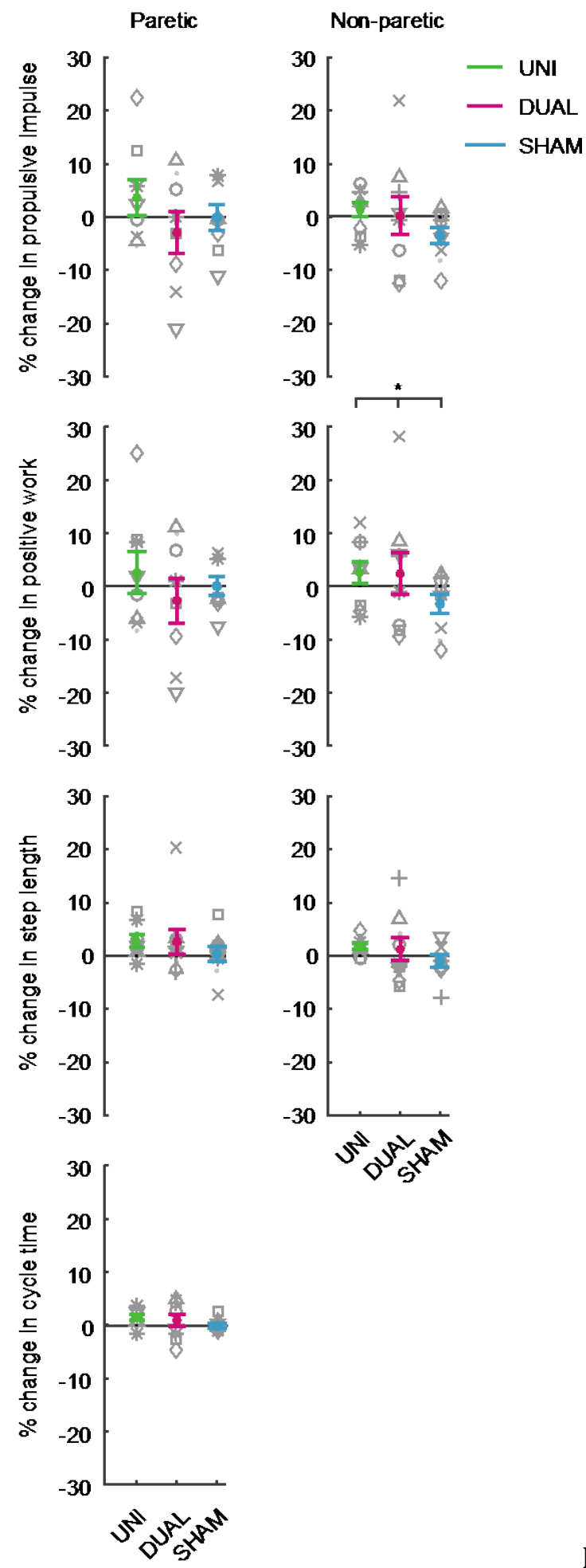

Figure 6. Group averages for the outcome variables for

the stroke survivors expressed as a percentage change from the baseline values for the different stimulation conditions. The thick dotted marker and error bars indicate the across subject averages and standard error of the mean. The gray markers indicate the individual responses (each subject has its own marker). 


\section{Tables}

Table 1: Characteristics of the chronic stroke survivors

\begin{tabular}{|l|l|l|l|l|l|l|l|l|l|l|l|}
\hline $\begin{array}{l}\text { Stroke } \\
\text { ID }\end{array}$ & gender & age & $\begin{array}{l}\text { time } \\
\text { since } \\
\text { stroke }\end{array}$ & $\begin{array}{l}\text { affected } \\
\text { hemisphere }\end{array}$ & stroke type & $\begin{array}{l}\text { stroke } \\
\text { location }\end{array}$ & FAC & MI & FM & Speed & SA \\
\hline 1 & female & 46 & 51 & right & hemorrhagic & subcortical & 5 & 91 & 25 & 1.35 & 0.04 \\
\hline 2 & male & 38 & 6 & right & ischemic & subcortical & 5 & 83 & 29 & 1.29 & -0.02 \\
\hline 3 & male & 62 & 27 & left & ischemic & subcortical & 4 & 39 & 18 & 0.42 & -0.14 \\
\hline 4 & female & 69 & 93 & left & ischemic & subcortical & 5 & 64 & 30 & 0.85 & -0.38 \\
\hline 5 & female & 68 & 86 & left & hemorrhagic & cortical & 5 & 64 & 26 & 0.72 & 0.33 \\
\hline 6 & male & 45 & 44 & left & ischemic & cortical & 5 & 75 & 28 & 1.03 & 0.13 \\
\hline 7 & male & 66 & 12 & right & ischemic & subcortical & 5 & 53 & 22 & 0.82 & -0.11 \\
\hline 8 & female & 62 & 11 & right & ischemic & cortical & 5 & 64 & 27 & 0.71 & 0.00 \\
\hline 9 & female & 71 & 12 & left & ischemic & cortical & 5 & 72 & 29 & 0.92 & 0.03 \\
\hline 10 & female & 53 & 105 & left & ischemic & cortical & 5 & 72 & 24 & 0.84 & 0.02 \\
\hline
\end{tabular}

Abbreviations: FAC, score on Functional Ambulation Categories; MI, score on leg portion of

motricity index; FM, score on leg portion of Fugl Meyer score; speed, walking speed on $10 \mathrm{~m}$ walking

test; SA, step length asymmetry defined as the difference between the paretic and non-paretic step

length divided by the sum of both. A positive number indicates a larger paretic than non-paretic step

length. 
Table 2: Across subject averages ( \pm standard deviation) of the variables from the non preferred leg of the healthy subjects for the different stimulation conditions (STIM) and the average differences ([95 $\%$ confidence interval]) between the different stimulation conditions. Averages are expressed as percentage change with respect to the baseline scores.

\begin{tabular}{|c|c|c|c|c|c|c|}
\hline & \multicolumn{3}{|c|}{$\begin{array}{l}\text { Average change (\%) per stimulation } \\
\text { condition } \\
\text { (average } \pm \text { standard deviation) }\end{array}$} & \multicolumn{3}{|c|}{$\begin{array}{l}\text { Difference between stimulation } \\
\text { conditions (average [95\% } \\
\text { confidence interval]]) }\end{array}$} \\
\hline & UNI & DUAL & SHAM & UNI-DUAL & $\begin{array}{l}\text { UNI- } \\
\text { SHAM }\end{array}$ & $\begin{array}{l}\text { DUAL- } \\
\text { SHAM }\end{array}$ \\
\hline \multicolumn{7}{|l|}{ Non preferred leg } \\
\hline $\begin{array}{l}\text { Propulsion } \\
\text { impulse }^{\dagger}\end{array}$ & $2.8 \pm 2.8$ & $4.4 \pm 9.1$ & $-0.7 \pm 6.5$ & $\begin{array}{l}-1.5 \\
{[-5.17 .2]}\end{array}$ & $\begin{array}{l}3.6 \\
{[0.07 .2]}\end{array}$ & $\begin{array}{l}5.1 \\
{[1.5 \text { 8.7] }}\end{array}$ \\
\hline Positive work ${ }^{\dagger}$ & $3.1 \pm 6.0$ & $4.7 \pm 9.4$ & $-0.5 \pm 6.8$ & $\begin{array}{l}-1.6 \\
{[-5.42 .2]}\end{array}$ & $\begin{array}{l}3.6 \\
{[-0.27 .4]}\end{array}$ & $\begin{array}{l}5.2 \\
{[1.49 .0]}\end{array}$ \\
\hline Step length ${ }^{\dagger}$ & $-0.1 \pm 3.3$ & $1.6 \pm 3.2$ & $0.1 \pm 2.9$ & $\begin{array}{l}-1.7 \\
{[-3.3-0.2]}\end{array}$ & $\begin{array}{l}-0.2 \\
{\left[\begin{array}{ll}-1,8 & 1.3\end{array}\right]}\end{array}$ & $\begin{array}{l}1.5 \\
{[-0.13 .1]}\end{array}$ \\
\hline Stance Duration $^{\dagger}$ & $0.2 \pm 2.7$ & $2.0 \pm 3.3$ & $0.0 \pm 2.7$ & $\begin{array}{l}-1.8 \\
{[-3.2-0.4]}\end{array}$ & $\begin{array}{l}0.2 \\
{\left[\begin{array}{ll}-1.2 & 1.6\end{array}\right]}\end{array}$ & $\begin{array}{l}2.0 \\
{[0.63 .4]}\end{array}$ \\
\hline Cycle time & $0.2 \pm 2.6$ & $2.0 \pm 3.3$ & $0.0 \pm 2.9$ & 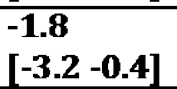 & $\begin{array}{l}0.2 \\
{[-1.2} \\
\end{array}$ & $\begin{array}{l}2.0 \\
{[0.63 .4]}\end{array}$ \\
\hline
\end{tabular}

t indicates significant main effect of STIM with $p<0.05$.

Bold font indicates a significant effect for the post-hoc comparisons, $\mathrm{p}<0.05$ 
Table 3. Across subject averages ( \pm standard deviation) of the variables from the paretic and non paretic leg of the stroke survivors for the different stimulation conditions (STIM) and the average differences ([95\% confidence interval] $)$ between the different stimulation conditions. Averages are expressed as percentage change with respect to the baseline scores.

\begin{tabular}{|c|c|c|c|c|c|c|}
\hline & \multicolumn{3}{|c|}{$\begin{array}{l}\text { Average change }(\%) \text { per stimulation } \\
\text { condition } \\
\text { (average } \pm \text { standard deviation) }\end{array}$} & \multicolumn{3}{|c|}{$\begin{array}{l}\text { Difference between stimulation } \\
\text { conditions (average [95\% } \\
\text { confidence interval]] }\end{array}$} \\
\hline & UNI & DUAL. & SHAM & UNI-DUAI. & $\begin{array}{l}\text { UNI- } \\
\text { SHAM }\end{array}$ & $\begin{array}{l}\text { DUAL- } \\
\text { SHAM }\end{array}$ \\
\hline \multicolumn{7}{|l|}{ Paretic } \\
\hline $\begin{array}{l}\text { Propulsion } \\
\text { impulse }\end{array}$ & $3.7 \pm 10.3$ & $-3.0 \pm 11.2$ & $-0.1 \pm 7.9$ & $\begin{array}{l}6.6 \\
{[-2.315 .5]}\end{array}$ & $\begin{array}{l}3.7 \\
{[-5.112 .6]}\end{array}$ & $\begin{array}{l}-2.9 \\
{[-11.76 .0]}\end{array}$ \\
\hline Positive work & $2.6 \pm 11.7$ & $-2.7 \pm 12.2$ & $0.1 \pm 7.7$ & $\begin{array}{l}5.3 \\
{\left[\begin{array}{ll}-4.5 & 15.0\end{array}\right]}\end{array}$ & $\begin{array}{l}2.5 \\
{\left[\begin{array}{lll}-7.2 & 12.3\end{array}\right]}\end{array}$ & $\begin{array}{l}-2.7 \\
{[-12.57 .0]}\end{array}$ \\
\hline Step length & $2.8 \pm 4.1$ & $2.6 \pm 7.6$ & $0.4 \pm 4.4$ & $\begin{array}{l}0.2 \\
{[-4.14 .5]}\end{array}$ & $\begin{array}{l}2.4 \\
{[-1.96 .7]}\end{array}$ & $\left.\begin{array}{ll}2.2 \\
{[-2.1} & 6.6\end{array}\right]$ \\
\hline Stance Duration & $1.1 \pm 2.8$ & $0.9 \pm 3.8$ & $0.2 \pm 1.5$ & $\begin{array}{l}0.1 \\
{\left[\begin{array}{ll}-1.9 & 2.2\end{array}\right]}\end{array}$ & $\begin{array}{l}1.1 \\
{[-1.03 .1]}\end{array}$ & $\begin{array}{l}0.9 \\
{[-1.13 .0]}\end{array}$ \\
\hline \multicolumn{7}{|l|}{ Non-paretic } \\
\hline $\begin{array}{l}\text { Propulsion } \\
\text { impulse }\end{array}$ & $1.4 \pm 5.5$ & $0.3 \pm 11.0$ & $-3.5 \pm 6.0$ & $\begin{array}{l}1.2 \\
{[-4.66 .9]}\end{array}$ & $\begin{array}{l}4.9 \\
{\left[\begin{array}{lll}-0.8 & 10.6\end{array}\right]}\end{array}$ & $\begin{array}{l}3.7 \\
{[-2.09 .5]}\end{array}$ \\
\hline Positive work $^{\dagger}$ & $2.7 \pm 6.8$ & $2.5 \pm 12.5$ & $-3.3 \pm 6.6$ & $\begin{array}{l}0.2 \\
{[-6.06 .4]}\end{array}$ & $\begin{array}{l}5.9 \\
{\left[\begin{array}{ll}-0.3 & 12.1\end{array}\right]}\end{array}$ & $\begin{array}{l}5.7 \\
{\left[\begin{array}{lll}-0.5 & 11.9\end{array}\right]}\end{array}$ \\
\hline Step length & $1.7 \pm 3.0$ & $1.3 \pm 7.1$ & $-1.0 \pm 4.0$ & $\begin{array}{l}0.4 \\
{[-3.84 .7]}\end{array}$ & $\begin{array}{l}2.7 \\
{\left[\begin{array}{lll}-1.6 & 6.9\end{array}\right]}\end{array}$ & $\begin{array}{ll}2.2 \\
{[-2.0} & 6.5]\end{array}$ \\
\hline Stance duration & $1.5 \pm 1.9$ & $0.9 \pm 3.9$ & $-0.1 \pm 1.8$ & $\begin{array}{l}0.6 \\
{\left[\begin{array}{ll}-1.5 & 2.7\end{array}\right]}\end{array}$ & $\begin{array}{l}1.6 \\
{\left[\begin{array}{ll}-0.5 & 3.7\end{array}\right]}\end{array}$ & $\begin{array}{l}1.0 \\
{[-1.13 .1]}\end{array}$ \\
\hline Cycle time & $1.5 \pm 1.8$ & $1.0 \pm 3.7$ & $-0.2 \pm 1.5$ & $\begin{array}{l}0.5 \\
{[-1.42 .4]}\end{array}$ & $\begin{array}{l}1.6 \\
{[-0.33 .6]}\end{array}$ & $\begin{array}{l}1.2 \\
{[-0.73 .1]}\end{array}$ \\
\hline
\end{tabular}

t indicates significant main effect of STIM with $p<0.05$. 